\title{
Big Bang: Uma Ferramenta para Auxiliar no Ensino-Aprendizagem da Classificação Periódica dos Elementos Químicos
}

\author{
Breno A. L. Andrade ${ }^{1}$, Pablo F. Matos ${ }^{1}$, Daiana F. L. Santos ${ }^{2}$, Wdson C. Santos ${ }^{2}$ \\ ${ }^{1}$ Bacharelado em Sistemas de Informação - Instituto Federal de Educação, Ciência e \\ Tecnologia da Bahia (IFBA) - Vitória da Conquista - BA - Brasil \\ ${ }^{2}$ Licenciatura em Química - Instituto Federal de Educação, Ciência e Tecnologia da \\ Bahia (IFBA) - Vitória da Conquista - BA - Brasil \\ breno.lessa.andradelgmail.com, pablofmatoseifba.edu.br, \\ daianaflores.12@hotmail.com, wdsoncosta@ifba.edu.br
}

\begin{abstract}
The educational software Big Bang aims to modernize and act as a facilitator tool in the learning environment, enabling easy interaction and learning of the students about the periodic table of the elements through contextualized tips that promote reasoning, making them learn dynamically and playful. Once the classroom environment needs to adapt to new ways of teaching, it is clear that the Big Bang is a promising application. It was applied a survey in order to classify the chemical elements by levels.
\end{abstract}

Resumo. O software educacional Big Bang visa modernizar e agir como ferramenta facilitadora no ambiente de ensino, possibilitando a fácil interação e aprendizagem dos estudantes sobre a classificação periódica dos elementos através de dicas contextualizadas que promovem o raciocínio, fazendo com que os mesmos aprendam de maneira dinâmica e lúdica. Uma vez que o ambiente da sala de aula necessita se adaptar as novas formas de ensino, constata-se que o Big Bang é uma aplicação promissora. Aplicou-se um questionário com o intuito de classificar os elementos químicos por níveis.

\section{Introdução}

Atualmente, muitas instituições como Feevale [Feevale 2015] e Unisul [Unisul 2015] e grupos de pesquisa (e.g., NIEE ${ }^{1}$, GEINFOEDU ${ }^{2}, \mathrm{GPIE}^{3}$ ) têm estudado e implantado os mais diversos conceitos de informática vinculada à educação. Nesse sentido, os estudantes adquirem habilidades cognitivas e intelectuais, o que faz que o mesmo desenvolva a capacidade de se tornar um ser autônomo, capaz de aprender e buscar novos conhecimentos. O computador torna-se, então, aliado, proporcionando novas formas de pensar, agir e assimilar o conhecimento.

Explorar os recursos computacionais faz com que haja uma melhora significativa no aprendizado e desenvolvimento do censo criativo do estudante. Um exemplo dos inúmeros recursos é os jogos que são reconhecidos como grande aliado para o ensino, conseguindo colocar o estudante próximo aos conteúdos e aproximar o educador e o educando. Necessita ser sempre voluntário, desafiante, motivador e provocador de curiosidade [Bardiviesso 2010].

\footnotetext{
${ }^{1}$ www.ufrgs.br/niee

$2 \mathrm{http}: / /$ porteiras.s.unipampa.edu.br/geinfoedu/

${ }^{3}$ www2.joinville.udesc.br/ gpie/site
} 
Os jogos, de modo geral, sempre estiveram presentes na vida das pessoas, seja como elemento de diversão, disputa ou como forma de aprendizagem. Por meio de sua análise em diferentes épocas, pode-se perceber que jogar sempre foi uma atividade inerente do ser humano [Cunha 2012]. Segundo [Shoreder and Pruner 2010], os jogos e as brincadeiras despertam no estudante o interesse pelas atividades escolares e auxiliam na construção do conhecimento. Cabe ao professor a articulação das atividades lúdicas com os conteúdos curriculares, o que proporciona aos estudantes momentos espontâneos e prazerosos na aquisição de aprendizagem.

Percebe-se a ausência de uma proposta pedagógica voltada para as atividades lúdicas nas escolas. Durante muito tempo, o brincar não estava relacionado à aprendizagem. Porém, ainda segundo [Shoreder and Pruner 2010], muitos estudos foram realizados sobre o assunto, e foi constatado que as brincadeiras, quando bem ministradas pelos educadores e relacionadas aos conteúdos curriculares, proporcionam aos estudantes um aprendizado prazeroso.

A partir deste contexto, percebe-se a necessidade de integração e modernização do ensino da química, para que esta deixe de ser apenas uma disciplina do ensino médio. O desejável é que os assuntos abordados comecem a fazer parte do cotidiano dos estudantes de maneira dinâmica e lúdica, despertando o interesse pelo aprendizado. Nesse sentido, este trabalho tem como principal objetivo apresentar o desenvolvimento do software educacional, denominado Big Bang, sendo este direcionado ao ensino e aprendizado da classificação periódica dos elementos químicos, As principais características do software educacional proposto são: (i) possui um sistema de pontuação focado no estímulo do estudante; (ii) possui níveis de dificuldade que visam à obtenção gradual de conhecimento e avanço no jogo; (iii) possui dicas elaboradas por estudiosos da área de química que instigam a curiosidade; e (iv) interface amigável.

O restante do artigo está estruturado da seguinte forma: na Seção 2 são apresentados os trabalhos correlatos; $\mathrm{Na}$ Seção 3 é discutida a metodologia de desenvolvimento; $\mathrm{Na}$ Seção 4 são apresentadas as características do software educacional Big Bang; Na Seção 5 é abordado como se procedeu a classificação dos elementos, através de aplicação e análise de questionário; E por fim, na Seção 6 são feitas algumas considerações finais e trabalhos futuros.

\section{Trabalhos Correlatos}

Os softwares educacionais são materiais novos e diferenciados que promovem contribuição na aprendizagem com motivação e tornam o ensino mais dinâmico. MyQuímica [Silva and Rebouças 2011], Advinhas da Tabela Periódica, Descoberta dos Pares Químicos e Borboletas Químicas [Paiva et al. 2015] são exemplos desse tipo de software. Porém, alguns destes trabalham especificamente com a aprendizagem das propriedades dos elementos químicos e no reconhecimento destes, e outros apresentam somente como meta o auxílio nas propriedades dos elementos. Entretanto, o software Big Bang foi criado com o objetivo de contemplar tanto o ensino das características e memorização dos elementos químicos quanto o estudo das propriedades periódicas.

Os jogos didáticos são utilizados por docentes como metodologia inovadora e atraente para contribuição do ensino de química. Um Super Trunfo [Godoi, Oliveira and Codognoto 2010], Ludo Químico [Costa 2013] e Bingo da Tabela Periódica [Oliveira et al. 2012], são modelos de materiais que foram aplicados em sala de aula para apoio do 
ensino das propriedades periódicas e resultaram de muita importância aos discentes, pois os mesmos demonstraram estimulo para realizarem as atividades.

O software Big Bang foi baseado em um jogo didático de tabuleiro para ser utilizado com os alunos do ensino médio, elaborado pela graduanda do curso de Licenciatura em Química Priscila Silva Carvalho, enquanto bolsista do PIBID. Este é composto por: (1) um tabuleiro (imagem da tabela periódica sem os elementos), contendo os períodos e famílias, devidamente numerados, o diagrama de Linus Pauling, e os subníveis mais energéticos; (2) carta elemento (CE) contendo o símbolo químico, número atômico e uma imagem representativa; (3) carta dica (CD) com três dicas sobre cada elemento; (4) fichas de pontuação. As CE são dispostas sobre a mesa, enquanto as CD são embaralhadas e colocadas com as dicas para baixo.

O jogo ocorre em três etapas: Etapa 1, o leitor tira uma carta e lê uma dica de cada vez; Etapa 2, uma vez descoberto qual o elemento, o jogador deve identificar a carta referente ao mesmo, através do seu símbolo químico; Etapa 3, utilizando o número atômico, e/ou a distribuição eletrônica, o jogador deve encontrar a posição em que o elemento se encontra na tabela, e fixá-lo no tabuleiro. Acertando o elemento com uma dica, o jogador marca 05 pontos, com duas 03 pontos, e com três apenas 01 ponto. Obtém mais 05 pontos pela identificação do símbolo e localização correta no tabuleiro. Assim, o software Big Bang foi elaborado através das modificações e adaptações, com o intuito de auxiliar o acesso à informação e estimular ao aprendiz de forma mais atrativa para contribuição no processo do ensino e aprendizagem [Souza, I. and Souza, L. 2010].

\section{Metodologia de Desenvolvimento}

A metodologia de desenvolvimento do software educacional Big Bang é composta por três etapas, a saber, (Figura 1): (1) Tecnologias, (2) Banco de Dados e (3) Implementação.

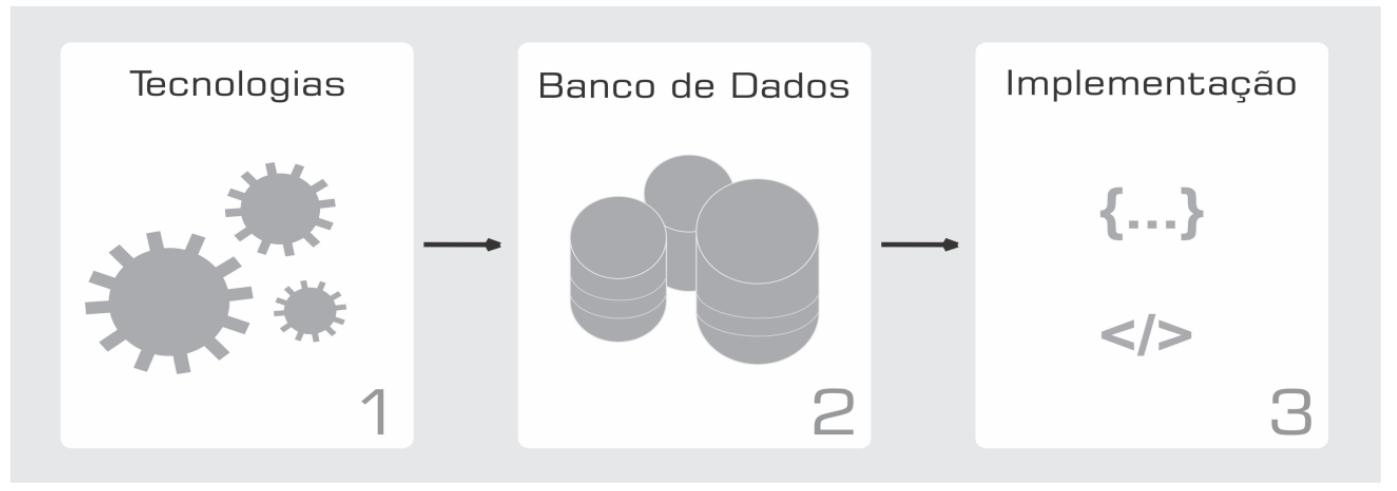

Figura 1. Síntese das Etapas Fonte. Próprio autor

Inicialmente foi feito um levantamento bibliográfico dos softwares educativos existentes, relacionados ao tema tabela periódica. Em seguida foi realizada uma análise a respeito da linguagem de programação a ser utilizada no desenvolvimento do software educativo. Posteriormente, foram criadas as regras e inserido os conceitos no software com a ajuda de uma estudante e um professor do curso de licenciatura em química. Após este procedimento, deu-se início o desenvolvimento do software educativo a partir dos conceitos e das regras definidas, juntamente com a base de dados com dicas acerca 
dos elementos da tabela periódica, no qual foi possível criar um sistema de dificuldade em níveis e ao mesmo tempo implantar o sistema de pontuação nas dicas.

\subsection{Tecnologias}

Na Etapa 1 é definida a plataforma WEB através do uso da linguagem HTML5, que é a nova versão da linguagem de marcação e apresentação de texto utilizada na WEB. Apresenta tags mais semânticas, dando um novo significado à WEB, além de recursos como o elemento Canvas, disponibilidade da API WebGL e tags específicas para vídeo e áudio [Silva 2011].

A principal característica que motivou a utilização do HTML5 é a possibilidade de uso do software em computadores e em dispositivos móveis de forma simples, sem que seja necessário instalar programas adicionais, além da facilidade em integrar recursos adicionais como: CSS3 para personalização e desenvolvimento de todo o layout do jogo; PHP para requisições ao banco de dados, uma vez que o HTML é uma linguagem de marcação, não conseguindo por si só realizar consultas a banco de dados; e, por fim, JavaScript que é uma linguagem de script executada no navegador do usuário, possibilitando acesso dinâmico às páginas WEB e ao mesmo tempo removendo as sobrecargas de processamento dos servidores de hospedagem do software educacional.

Nesse sentido, a escolha da tecnologia utilizada para o desenvolvimento do software foi o HTML5 puro devido às vantagens listadas anteriormente e à possibilidade de ter acesso e controle total do código fonte, diferente de uma game engine como o Construct $2^{4}$, o Unity $3 \mathrm{D}^{5}$ e o Unreal Engine $4^{6}$ que abstrai algumas partes do desenvolvimento.

\subsection{Banco de Dados}

$\mathrm{Na}$ Etapa 2 é projetado o banco de dados, onde serão armazenados todos os dados e as características do jogo. A ferramenta de modelagem de dados utilizada para desenvolver os três esquemas (conceitual, lógico e físico) foi o brModelo [Cândido 2005]. A modelagem conceitual baseia-se no mais alto nível e deve ser usada para envolver o cliente, pois o foco é discutir os aspectos do negócio do cliente e não da tecnologia. $\mathrm{O}$ esquema conceitual é uma descrição concisa dos requisitos de dados dos usuários e inclui detalhes dos tipos de entidade, relacionamentos e restrições. Como não incluem descrições detalhadas de implementação, esses conceitos normalmente são mais fáceis de entender [Elmasri and Navathe 2011].

O esquema lógico define os tipos de dados, as chaves primárias e estrangeiras, normalização, integridade referencial, entre outras. Para o esquema lógico ser criado, leva-se em conta a modelagem de dados criada no modelo conceitual.

No esquema físico deve-se escolher um Sistema de Gerenciamento de Banco de Dados (SGBD), e realizar a modelagem física do esquema de banco de dados. A modelagem física nada mais é do que recorrer a linguagem SQL para implementar o que foi definido no modelo lógico. O SGBD MySQL foi o escolhido por possuir

\footnotetext{
${ }^{4}$ https://www.scirra.com/construct2

${ }^{5} \mathrm{http}: / /$ unity3d.com/

${ }^{6} \mathrm{https}: / /$ www.unrealengine.com/what-is-unreal-engine-4
} 
características que preenchem os requisitos necessários para a aplicação, por exemplo: open source, possui consistência, alta performance, confiabilidade, simples de manusear, além disso, possui uma comunidade ativa e disposta a solucionar problema que possam surgir na etapa de desenvolvimento [MySQL 2015].

Na elaboração da base de dados ocorreu a seleção dos elementos químicos mais comuns, com dicas relacionadas a estes e as propriedades periódicas. A elaboração da base de dados foi realizada por um discente de Licenciatura em Química, bolsista do projeto PIBID (Programa Institucional de Bolsa de Iniciação à Docência), e um docente da mesma área, por apresentarem conhecimentos específicos na área de atuação do jogo.

\subsection{Implementação}

Na Etapa 3 é implementado o software educacional. Sendo nesta etapa que ocorre a integração dos componentes gráficos (HTML, CSS e imagens) com a lógica proporcionada pelo JavaScript. Foi utilizada a ferramenta Inkscape [The Inkscape Team 2015], selecionada para construção de alguns componentes da parte gráfica do jogo (e.g., estrutura do átomo, avatares e troféus), justificando sua escolha principalmente pelo fato dos integrantes do grupo apresentarem conhecimentos prévios. Outro ponto a destacar é o uso do PHP [Soares 2010], no qual foi responsável por gerenciar o acesso ao banco de dados.

\section{Big Bang}

Big Bang $^{7}$ (Figura 2) é um software educacional de raciocínio, que envolve e estimula o estudante por meio de dicas que associam os elementos químicos a características e atividades cotidianas, para que o mesmo descubra o elemento químico em questão e os demais assuntos relacionados (organização da tabela e distribuição eletrônica). O intuito é fazer com que o estudante aprenda de maneira dinâmica e lúdica. O nome Big Bang foi adotado para o software por ser o nome da teoria de formação do universo. Apresenta relação com o tema do jogo (tabela periódica) por envolver elementos químicos, que estão presentes na tabela e, por sua vez, estão presentes na formação do universo.

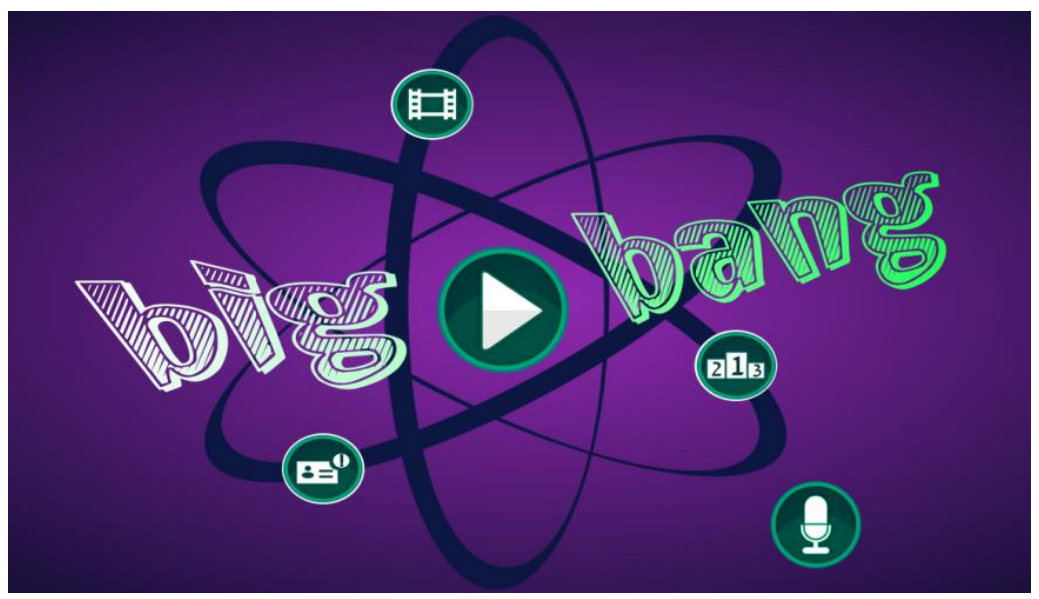

Figura 2. Tela Inicial do Jogo Big Bang Fonte. Próprio autor

\footnotetext{
${ }^{7}$ http://gse.conquista.ifba.edu.br/softwares/\#bigbang
} 


\subsection{Dicas}

O sistema de dicas do software educativo possui as seguintes características: o jogador recebe uma determinada pontuação de acordo com a quantidade de dicas utilizadas para acertar o elemento químico; quanto mais dicas utilizar menos pontos acumulará; está disponível para cada elemento químico do jogo até 03 (três) dicas para serem utilizadas pelo jogador. Um exemplo de dicas pode ser encontrado na Tabela 1. É importante salientar que alguns elementos apresentam propriedades semelhantes e, consequentemente, algumas dicas são semelhantes, devendo o jogador utilizar as demais dicas restantes e ao mesmo tempo conhecimentos adquiridos em sala de aula para acertar o elemento.

Tabela 1. Exemplo de Dicas do Nível Iniciante

\begin{tabular}{|c|l|l|}
\hline$\#$ & \multicolumn{1}{|c|}{ Dica } & \multicolumn{1}{|c|}{ Pontuação } \\
\hline 1 & Presente nos ossos. & 05 pontos \\
\hline 2 & Se consumido em excesso, causa pedra nos rins. & 03 pontos \\
\hline 3 & Composição do mármore. & 01 ponto \\
\hline
\end{tabular}

Fonte. Próprio autor

\subsection{Banco de Dados}

As dicas do banco de dados são criadas para o reconhecimento de algumas propriedades, aplicações e classificação periódicas dos elementos químicos. As dicas requerem conhecimento referente à tabela periódica, suas propriedades e estão relacionadas com algum elemento.

Ao todo foram selecionados 45 (quarenta e cinco) elementos e elaboradas 135 (cento e trinta e cinco) dicas, sendo três destas para cada elemento. Os elementos estão separados em três níveis (iniciante, curioso e cientista), sendo 15 (quinze) elementos para cada nível. Cada vez que se inicia o jogo faz-se uma seleção aleatória de 12 (doze) elementos químicos, sendo 08 (oito) de fato utilizados em cada rodada. Os 04 (quatros) elementos sobressalentes são para dar rotatividade aos elementos em cada rodada e para não ficar óbvio a seleção do último elemento.

As cartas com os elementos possuem apenas o número atômico e uma imagem representando os mesmos. Acertando a dica, o jogador obtém a pontuação de acordo com a quantidade de dicas que foram necessárias para o seu acerto e marca mais 05 pontos pelo posicionamento correto na tabela. Com isso, envolve tanto a identificação do elemento químico, quanto os conhecimentos sobre organização da tabela e sua relação com a distribuição eletrônica.

No diagrama da Figura 3 é apresentado o esquema conceitual de dados do software educacional Big Bang. Como pode-se perceber, há quatro (04) entidades no esquema conceitual, a saber: "ranking", "nivel", "questao" e "dicas". A entidade "ranking" é utilizada para armazenar os dados do nome usuário, o nível do jogo, a pontuação e a data/hora que o jogador estava jogando, além do código identificador; a entidade "nivel" armazena o código único e o nome do nível; a entidade "questao" armazena um código identificador e a resposta, que no caso, é o nome do elemento em questão no momento do jogo; e, por fim, a entidade "dicas" armazena as dicas para cada elemento, assim como, a sua pontuação e um código identificador. 

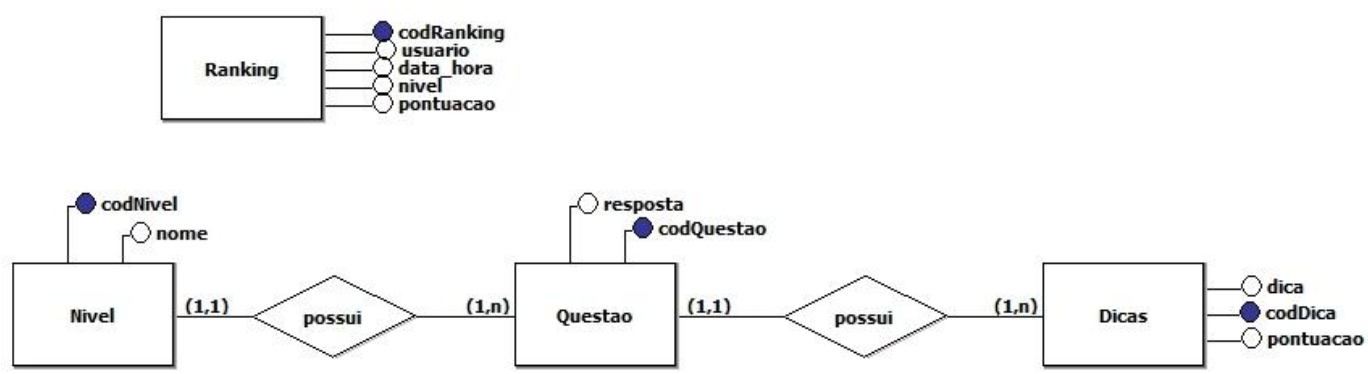

Figura 3. Esquema Conceitual Fonte. Próprio autor

\subsection{Características}

Foi definido um sistema de dificuldade em 03 (três) diferentes níveis (iniciante, curioso e cientista), a fim de estimular o participante a avançar no jogo. No Nível Iniciante, 05 (cinco) fatores são características deste nível: 1) Diagrama de Linus Pauling para realização da distribuição eletrônica; 2) Identificação do subnível mais energético de cada grupo; 3) Numeração da tabela de acordo as famílias; 4) Dicas pontuais sobre o elemento químico em questão; e 5) Distribuição eletrônica pela convenção cerne do gás nobre. No Nível Curioso, 03 (três) são os fatores que fazem parte deste nível: 1) Diagrama de Linus Pauling para realização da distribuição eletrônica; 2) Numeração da tabela de acordo as famílias; e 3) Dicas pontuais; No Nível Cientista, apenas 02 (dois) fatores, a saber: 1) Diagrama de Linus Pauling; e 2) Dicas pontuais. Como fator predominante deste último nível, espera-se do jogador um conhecimento apurado sobre os elementos químicos menos abordados no cotidiano. O jogador deverá selecionar e posicionar o elemento químico corretamente na tabela periódica, após a identificação do elemento a partir da dica informada. O resumo das características dos três níveis pode ser visualizado na Tabela 2.

Tabela 2. Sistema de Dificuldade em Níveis.

\begin{tabular}{|c|l|}
\hline Nível & \multicolumn{1}{c|}{ Característica } \\
\hline \multirow{5}{*}{ Iniciante } & $\begin{array}{l}\text { 1) Diagrama de Linus Pauling; } \\
\text { 2) Identificação do subnível mais energético; } \\
\text { 3) Numeração das famílias; } \\
\\
\text { 4) Dicas pontuais; } \\
\text { 5) Distribuição eletrônica pela convenção cerne do gás nobre. }\end{array}$ \\
\hline \multirow{2}{*}{ Curioso } & $\begin{array}{l}\text { 1) Diagrama de Linus Pauling; } \\
\text { 2) Numeração das famílias; } \\
\text { 3) Dicas pontuais. }\end{array}$ \\
\hline \multirow{2}{*}{ Cientista } & 1) Diagrama de Linus Pauling; \\
& 2) Dicas pontuais. \\
\hline
\end{tabular}

Fonte. Próprio autor

Um exemplo de tela no Nível Iniciante pode ser visualizado na Figura 4. O cenário é composto pela tabela periódica com a identificação do subnível energético característico de cada grupo, da distribuição eletrônica pela convenção cerne do gás nobre e do Diagrama de Linus Pauling, além do sistema de dicas e do posicionamento dos elementos químicos (imagem e símbolo atômico). Apresenta também o nome do jogador, um avatar escolhido pelo estudante/jogador antes de iniciar a rodada e a sua pontuação. Para pontuar o jogador deverá validar duas condições: (1) O elemento químico em questão a partir das dicas informadas e selecioná-lo. (2) Posicionar corretamente o elemento químico na tabela periódica. Caso o jogador acerte, será 
incrementada a pontuação. Caso contrário, será emitida uma mensagem de erro com um aviso sonoro.

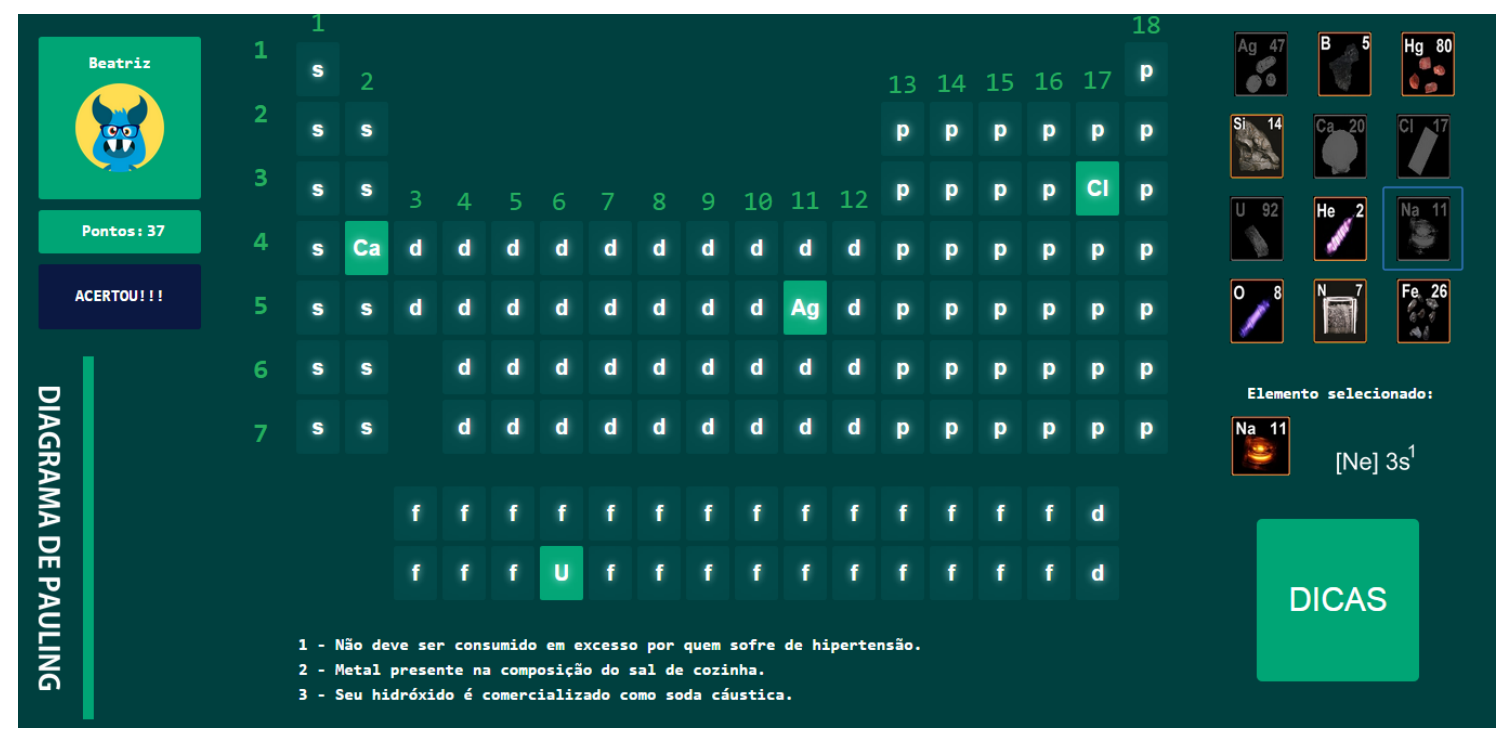

Figura 4. Tela Nível Iniciante Fonte. Próprio autor

Os 10 (dez) participantes com mais pontos entram no ranking de cada nível de classificação, sendo o primeiro lugar classificado pela maior pontuação. O sistema de pontuação possui a finalidade de incentivar o estudante a continuar jogando, que, por sua vez, o motivará a buscar a absorver mais conhecimento. O software educativo em questão possibilita que o estudante assimile conteúdos sobre: Classificação periódica dos elementos; propriedades, origem e aplicações dos elementos; distribuição eletrônica e sua relação com a organização da tabela periódica.

\section{Classificação dos Elementos}

Aplicou-se um questionário com o intuito de classificar os elementos químicos nos respectivos níveis (iniciante, curioso e cientista). O objetivo do questionário é avaliar o nível de dificuldade das questões que compõem o banco de dados do software educacional Big Bang. Para participar da avaliação foi selecionada uma turma com 25 (vinte e cinco) discentes do $3^{\circ}$ ano do Curso Técnico em Informática Integrado ao Ensino Médio.

O questionário é composto pelas dicas dos elementos. De acordo com estas, os discentes responderam quais eram os elementos referentes. Para a realização do questionário foi preciso uma duração de duas horas. Este tempo foi dividido em três etapas: primeiro, os discentes responderam ao questionário composto somente pelas primeiras dicas de cada um dos 45 (quarenta e cinco) elementos; depois responderam contendo a primeira e a segunda dica; e por último, responderam contendo as três dicas de cada elemento. Sendo realizado da mesma forma como apresentado no jogo online.

Para a avaliação do questionário foram analisadas as respostas da mesma forma como ocorre no jogo, quando se obteve o acerto com a primeira dica, foram atribuídos 05 pontos para o elemento, na segunda dica 03 pontos e na terceira 01 ponto. Para selecionar os elementos nos níveis foi preciso dividir o total dos elementos (45) pelo 
total dos níveis no jogo (03). Desta maneira, cada nível contém 15 (quinze) elementos. $\mathrm{Na}$ Tabela 3 é possível visualizar os elementos classificados nos seus respectivos níveis.

Para serem inseridos nos níveis, os elementos foram organizados em ordem crescente de pontuação. Com a classificação dos elementos nos níveis é possível observar que o elemento Hélio (He) obteve o número máximo de pontos, pois todos os discentes acertaram com a primeira dica, estando classificado no nível iniciante (fácil). Já o Zinco (Zn) apresenta o número mínimo de pontuação, pois obteve apenas dois acertos, sendo um destes 03 pontos com a segunda dica e 01 ponto com a última, totalizando 04 pontos, estando inserido no nível cientista (difícil).

Tabela 3. Classificação dos elementos em cada nível

\begin{tabular}{|c|c|c|c|c|c|}
\hline \multicolumn{2}{|c|}{ Cientista } & \multicolumn{2}{c|}{ Curioso } & \multicolumn{2}{c|}{ Iniciante } \\
\hline Elemento & Pontuação & Elemento & Pontuação & Elemento & Pontuação \\
\hline $\mathrm{Zn}$ & 04 & $\mathrm{Ac}$ & 28 & $\mathrm{Cu}$ & 53 \\
\hline $\mathrm{Br}$ & 15 & $\mathrm{Fr}$ & 28 & $\mathrm{U}$ & 58 \\
\hline $\mathrm{Ba}$ & 15 & $\mathrm{La}$ & 29 & $\mathrm{Hg}$ & 60 \\
\hline $\mathrm{Po}$ & 17 & $\mathrm{Ra}$ & 30 & $\mathrm{~B}$ & 64 \\
\hline $\mathrm{Sn}$ & 18 & $\mathrm{I}$ & 33 & $\mathrm{Cl}$ & 64 \\
\hline $\mathrm{Ti}$ & 19 & $\mathrm{Mg}$ & 36 & $\mathrm{Ag}$ & 64 \\
\hline $\mathrm{Pm}$ & 19 & $\mathrm{Cs}$ & 36 & $\mathrm{Ca}$ & 70 \\
\hline $\mathrm{Pb}$ & 19 & $\mathrm{Pt}$ & 40 & $\mathrm{~N}$ & 73 \\
\hline $\mathrm{Cr}$ & 19 & $\mathrm{~W}$ & 44 & $\mathrm{O}$ & 76 \\
\hline $\mathrm{Os}$ & 21 & $\mathrm{~F}$ & 45 & $\mathrm{Fe}$ & 79 \\
\hline $\mathrm{H}$ & 21 & $\mathrm{~K}$ & 45 & $\mathrm{Si}$ & 88 \\
\hline $\mathrm{Mn}$ & 23 & $\mathrm{Li}$ & 46 & $\mathrm{C}$ & 88 \\
\hline $\mathrm{Ni}$ & 24 & $\mathrm{Ne}$ & 48 & $\mathrm{Na}$ & 102 \\
\hline $\mathrm{Kr}$ & 25 & $\mathrm{Al}$ & 51 & $\mathrm{Au}$ & 106 \\
\hline $\mathrm{P}$ & 25 & $\mathrm{~S}$ & 51 & $\mathrm{He}$ & 125 \\
\hline
\end{tabular}

\section{Considerações Finais e Trabalhos Futuros}

Com base nos estudos realizados e o levantamento dos trabalhos correlatos, conclui-se que é necessário o desenvolvimento de softwares educacionais que seja capaz de fornecer diversão e ao mesmo tempo ensinar aos estudantes sobre a classificação periódica. Com isso, este trabalho objetivou apresentar as etapas do desenvolvimento do software educacional Big Bang, que tem o intuito de auxiliar a aprendizagem dos conteúdos relacionados à Tabela Periódica, utilizando o lúdico para facilitar o processo de ensino-aprendizagem.

Como trabalhos futuros, pretende-se aplicar o jogo em turmas de alunos do ensino médio, possibilitando medir o grau de satisfação ao jogar, se o mesmo é atraente, divertido e estimulante aos estudantes e se o software viabiliza o fácil aprendizado dos elementos químicos.

\section{Agradecimentos}

Os autores agradecem o apoio financeiro das agências de fomento à pesquisa do Brasil IFBA/PIBIC e CAPES/PIBID, e a participação de discentes que contribuíram positivamente com a resposta do questionário. 


\section{Referências}

Bardiviesso, A.M. (2010) O Lúdico na Matemática: Aprendendo com Jogos. $34 \mathrm{f}$. TCC (Licenciatura em Matemática) - Universidade Estadual de Mato Grosso do Sul, Cassilândia.

Cândido, C.H. (2005) Aprendizagem em Banco de Dados: Implementação de Ferramenta de Modelagem E.R. 44 f. Monografia (Especialização em Banco de Dados) - Universidade Federal de Santa Catarina e UNIVAG, Várzea Grande-MT.

Costa, A.F. (2013) Ludo Químico: Uma Alternativa Didática para o Ensino da Tabela Periódica. In: IX CONGIC, pages 1936-1943. IFRN.

Cunha, M.B. (2012) Jogos no ensino de química: considerações teóricas para sua utilização em sala de aula. Química Nova na Escola, 1, 34, 92-98.

Elmasri, R. and Navathe, S.B. (2011) Sistemas de Banco de Dados. Pearson Education, São Paulo.

Feevale. (2015) Informática na Educação, www.feevale.br/pesquisa/grupos-depesquisa/informatica-na-educacao.

Godoi, T.A.F., Oliveira, H.P.M. and Codognoto, L. (2010) Tabela Periódica: Um Super Trunfo para Alunos do Ensino Fundamental e Médio. Química Nova na Escola, 32, $1,22-25$.

MySQL. (2015) MySQL Forums, http://forums.mysql.com.

Oliveira, D.R.M. et al. (2012) Bingo da Tabela Periódica: Uma Atividade Lúdica Envolvendo Símbolos e Nomes dos Elementos. In: VII CONNEPI, pages 1-5. IFTO.

Paiva, J. et al. (2015) Jogos de Química: Advinhas da Tabela Periódica, Descoberta dos Pares Químicos e Borboletas Químicas, http://pfarma.com.br/jogos.html.

Shoreder, M.K. and Pruner, E.R. (2010) A importância das atividades lúdicas no processo ensino-aprendizagem. Pleiade, 7, 7, 108-115.

Silva, D.L.M. and Rebouças, A.D.D.S. (2011) MyQuímica: Um Jogo para Auxiliar no Ensino-Aprendizagem das Nomenclaturas Químicas. In XXII SBIE - XVII WIE. pp. 1858-1862.

Silva, M.S. (2011) HTML5: A Linguagem de Marcação que Revolucionou a Web. Novatec, São Paulo.

Soares, W. (2010) PHP 5: Conceitos, Programação e Integração com Banco de Dados. Érica, São Paulo.

Souza, I.M.A. and Souza, L.V.A. (2010) O Uso da Tecnologia como Facilitadora da Aprendizagem do Aluno na Escola. Revista Fórum Identidades, 8, 4, 127-142.

The Inkscape Team. (2015) Inkscape - Draw Freely, www.inkscape.org/pt.

Unisul. (2015) Grupo de Pesquisa em Informática na Educação, http://goo.gl/YgihkT. 